\title{
A Comparative Study on Loadings of the Lower Extremity during Deep Squat in Asian and Caucasian Individuals via OpenSim Musculoskeletal Modelling
}

\author{
Yichen Lu, ${ }^{1,2}$ Qichang Mei $\mathbb{D},{ }^{3,4}$ Hsien-Te Peng, ${ }^{1,5}$ Jianshe Li, ${ }^{3,4}$ Chen Wei $\mathbb{D},^{2}$ \\ and Yaodong $\mathbf{G u}^{3,4}$ \\ ${ }^{1}$ Graduate Institute of Sport Coaching Science, Chinese Culture University, Taipei 11114, Taiwan \\ ${ }^{2}$ Zhejiang Pharmaceutical College, Ningbo 315211, Zhejiang, China \\ ${ }^{3}$ Faculty of Sports Science, Ningbo University, Ningbo 315211, Zhejiang, China \\ ${ }^{4}$ Research Academy of Grand Health, Ningbo University, Ningbo 315211, Zhejiang, China \\ ${ }^{5}$ Department of Physical Education, Chinese Culture University, Taipei 11114, Taiwan
}

Correspondence should be addressed to Chen Wei; weichen_zj@hotmail.com

Received 4 February 2020; Revised 21 May 2020; Accepted 10 June 2020; Published 4 July 2020

Academic Editor: Prescott B. Chase

Copyright (c) 2020 Yichen Lu et al. This is an open access article distributed under the Creative Commons Attribution License, which permits unrestricted use, distribution, and reproduction in any medium, provided the original work is properly cited.

Populations of different ethnicities may present different movement capacities and muscular function adaptations. The purpose of this study was to investigate the differences of motion and loading in the lower extremity during dynamic deep squats between Asian and Caucasian individuals using OpenSim modelling technique with a customized squat-specific musculoskeletal model. Twenty-four participants joined the test performing the step-squat test, with right foot stepping side, squatting, and returning. The one-dimensional statistical parametric mapping package was used for statistical analysis. The main findings of the current study were as follows: (1) significant lower squat depth was observed in the Asian individuals, (2) the greater knee range of motion and contact forces were found in the Asian individuals, and (3) the greater ankle contact forces in the Caucasian individuals were notable while performing the deep squat compared to the Asian group. Knowledge found in the current study may provide implication for exercise practitioners and physiotherapists while designing schemes for the prevention of loading accumulation in the lower extremity.

\section{Introduction}

Squat is a motion practiced with synchronous ankle dorsiflexion, knee flexion, and hip flexion. It is a basic movement while performing functional activities in daily life, exercise training, or working environment. The squat attracted attention from the research fields of ethnics [1], occupation [2], clinics [3], biomechanics [4], and sports training $[1,5]$. The squat movement was considered an effective practice to strengthen the musculature of the lower extremity and trunk for the activation and coordination of a human motor system to maintain balance and stability. Thus, it has been extensively applied in clinical assessments [6] and even in microgravity conditions [7].

Squat depths and stance widths were reported to present different joint kinematics, kinetics, and muscle activities
[8-10]. Due to the altered distribution of biomechanical loadings, different squatting movements may be related to several malfunctions in the lower extremity, especially the knee joint. A review study reported that the squat can be generally categorized into a partial squat (around $40^{\circ}$ knee flexion), half squat (around $70-100^{\circ}$ knee flexion), and deep squat (over $100^{\circ}$ knee flexion) as the knee joint flexion angles are varying [11]. Nevertheless, recent studies defined knee flexion around $120 \sim 130^{\circ}$ as a deep squat $[12,13]$ aiming at quantifying knee loading and developing potential assistive devices for external support force of the knee joint.

Populations of different ethnicities may present different movement capacities and muscular function adaptations [1]. Previous researchers found that Asians presented a different squatting movement pattern [14], which might be a potential 
cause of the incidence of knee osteoarthritis [3]. However, information of a counterpart Caucasian group was not included in the previous study [14]. Furthermore, a recent study reported that using standard model simulations may overestimate loading while comparing to instrumented knee prostheses, and it was considered overestimated muscle moment arms, especially during large hip and knee joint motions [15]. Thus, a customized model for a maximal squat was adjusted and validated via altering the wrapping surface of the hip and knee muscles in a recent study [16].

The current study utilized an OpenSim musculoskeletal modelling technique [17] with a customized squat-specific model [16] and recruited Asian and Caucasian population cohorts to perform dynamic deep squats. It was aimed at investigating the differences in motion and loading in the lower extremity existing between Asian and Caucasian individuals. We hypothesized that the Asian individuals would have higher loading in the knee joint compared to the Caucasian individuals, while the Asian group may present a large range of motion in the knee joint.

\section{Materials and Methods}

2.1. Participants. A total of 24 participants were recruited into the study, including 12 Caucasian (9 males and 3 females, age: $27.4 \pm 4.8$ yrs, mass: $71.2 \pm 4.5 \mathrm{~kg}$, height: 1.73 $\pm 0.04 \mathrm{~m}$, BMI: $\left.23.78 \pm 2.49 \mathrm{~kg} / \mathrm{m}^{2}\right)$ and 12 Asian (8 males and 4 females, age: $27 \pm 3.7 \mathrm{yrs}$, mass: $65.4 \pm 6.9 \mathrm{~kg}$, height: $1.7 \pm 0.05 \mathrm{~m}$, BMI: $22.63 \pm 3.6 \mathrm{~kg} / \mathrm{m}^{2}$ ). All had history of physical exercise in either the gym or outdoors at least three times per week and 30 minutes each time. Specifically, the Caucasian group has an average time of 20 min per week performing "knee-not-past-toes" squat while the Asian group performed maximal (depth) squat for $15 \mathrm{~min}$ per week. The limb dominance was checked and defined as the right side via the preference of ball kicking. All participants were physically active without lower extremity disorders or injuries in the past six months prior to the test. They were informed of the objectives and requirements of this study with written consent. The testing protocol was approved by the ethics committee from the research institute.

2.2. Experimental Protocol. An eight-camera Vicon motion capture system (Vicon Metrics Ltd., Oxford, United Kingdom) and an embedded AMTI force plate (AMTI, Watertown, MA, United States) were synchronously used to record marker trajectories and ground reaction forces at a frequency of $200 \mathrm{~Hz}$ and $1000 \mathrm{~Hz}$, respectively. A previously established 37-marker set $[18,19]$ was used to trace the motion in the lower extremity. A surface Trigno wireless electromyography (EMG) measurement system (Delsys, Boston, MA, United States) was used to record the muscle activities. Muscle activities, including vastus lateralis (VL), vastus medialis (VM), biceps femoris (BF), semitendinosus (ST), tibialis anterior (TA), medial gastrocnemius (MG), and lateral gastrocnemius (LG), were collected with a frequency of $1000 \mathrm{~Hz}$. Maximal voluntary contractions (MVC) of the muscles were performed for the normalization of muscle activity (0-100\%) following a previously established protocol
[20]. Specifically, the single leg heel rise was performed to record maximal gastrocnemius activity, the seated knee extension against posterior resistance for knee extensors and the seated knee flexion against anterior resistance for knee flexors, and the supine hip flexion against inferior resistance and extension against superior resistance. The raw EMG signals were firstly filtered using a band-passed fourth order Butterworth filter between frequencies of $100-500 \mathrm{~Hz}$ in the Delsys EMGworks Analysis software. All processed signals ran the amplitude analysis with root mean square (RMS) calculation and then exported the processed values in the MVCs and activities from each trial. The EMG activity was calculated via the trial RMS amplitude values/MVC RMS amplitude values, as a zero ( 0 , no activation) to one ( $100 \%$, totally activated) scale, which was presented in our previous study [19].

Prior to the experiment, participants were instructed to perform a 10-minute warm up and practice the squatting movement. Specifically, the squatting movement started with (1) the right foot "stepping" aside onto the force platform, (2) maximal deep squat with the full foot contacting the force platform without lifting heel, and (3) then stepping off the platform. In the experiment, the marker position of the participant's static standing posture was initially recorded with legs being shoulder-width apart. During the deep squat test, participants initiated the squatting movement by side stepping onto the force platform (defined from the threshold of $20 \mathrm{~N}$ in the vertical ground reaction force), with the both legs (foot) naturally on stance width, without adjustments of foot orientation. Once the right foot was in full contact with the force platform, the maximal squat was performed until the deepest position of the pelvis, then returning to a standing position and finally stepping off the force platform. The squatting stance was defined by the right foot on and off the force platform with a $20 \mathrm{~N}$ threshold in the vertical ground reaction force. Three trials of the deep squat were collected for each participant.

2.3. Musculoskeletal Model. An OpenSim musculoskeletal model specifically customized for a squatting task [16] was used in this study. This model updated the original full body model $[17,18]$ by adjusting the wrapping surface at the knee and hip joints to allow high joint range of motion (ROM) [16], which has been validated via comparing simulated muscle activation against surface EMG signals. Although a full body model was used in this study, the interest was focused on the lower extremity, including a ball-and-socket hip joint with three degrees of freedom, a hinge knee joint (one degree of freedom) with a prescribed nonfrictional patella to direct the quadriceps muscle arm and a pin ankle joint (one degree of freedom). The subtalar and metatarsophalangeal joints were locked throughout data processing.

Marker trajectories and ground reaction forces were filtered at $12 \mathrm{~Hz}$ and $60 \mathrm{~Hz}$, respectively, prepared for data processing in OpenSim v3.3. A standard OpenSim workflow was conducted following an established protocol [17]. Firstly, the static marker position and body mass of each participant were used to scale the generic model and achieving a participant-matched model with muscle-tendon insertion and moment arms (Figure 1(a)). The scaling was conducted 


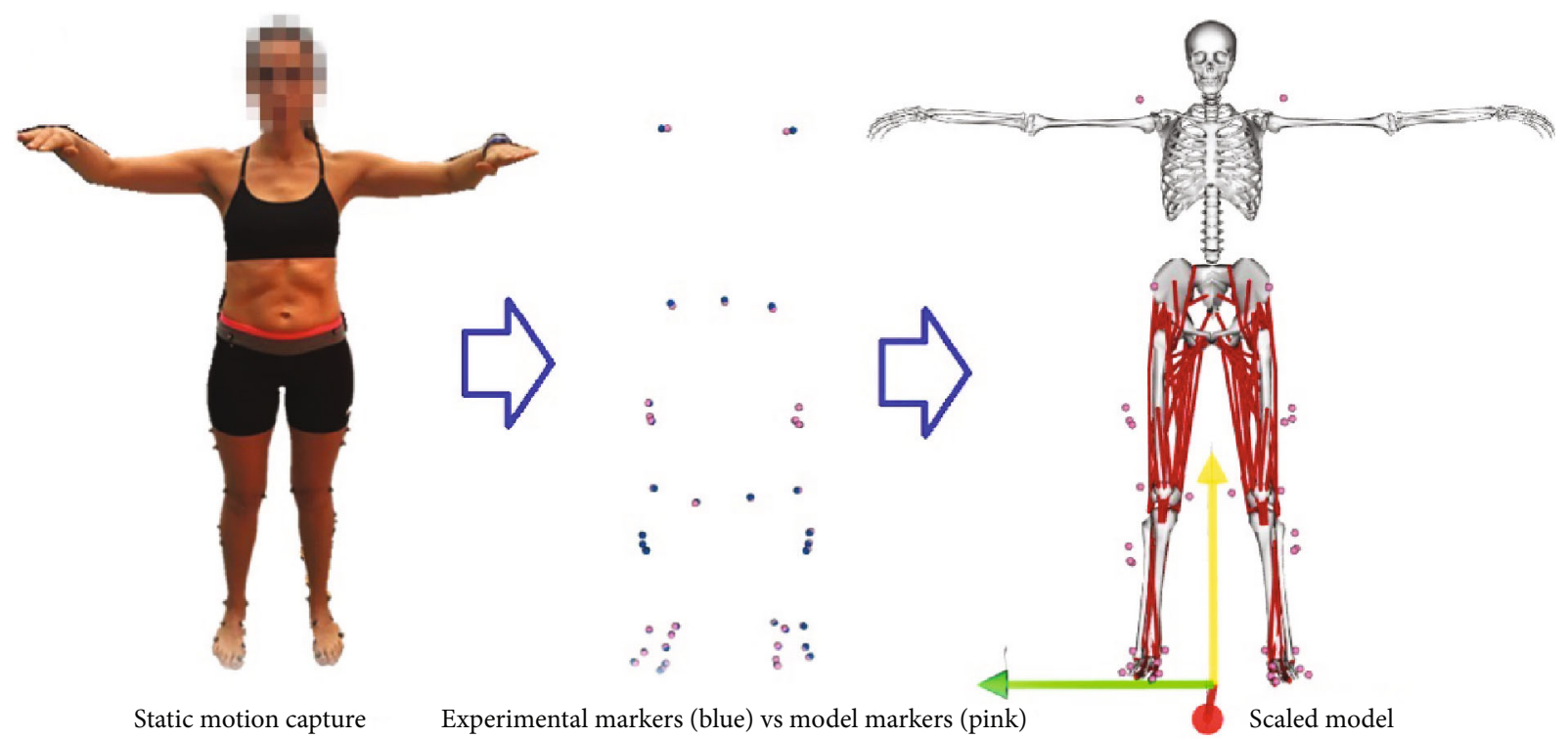

(a)
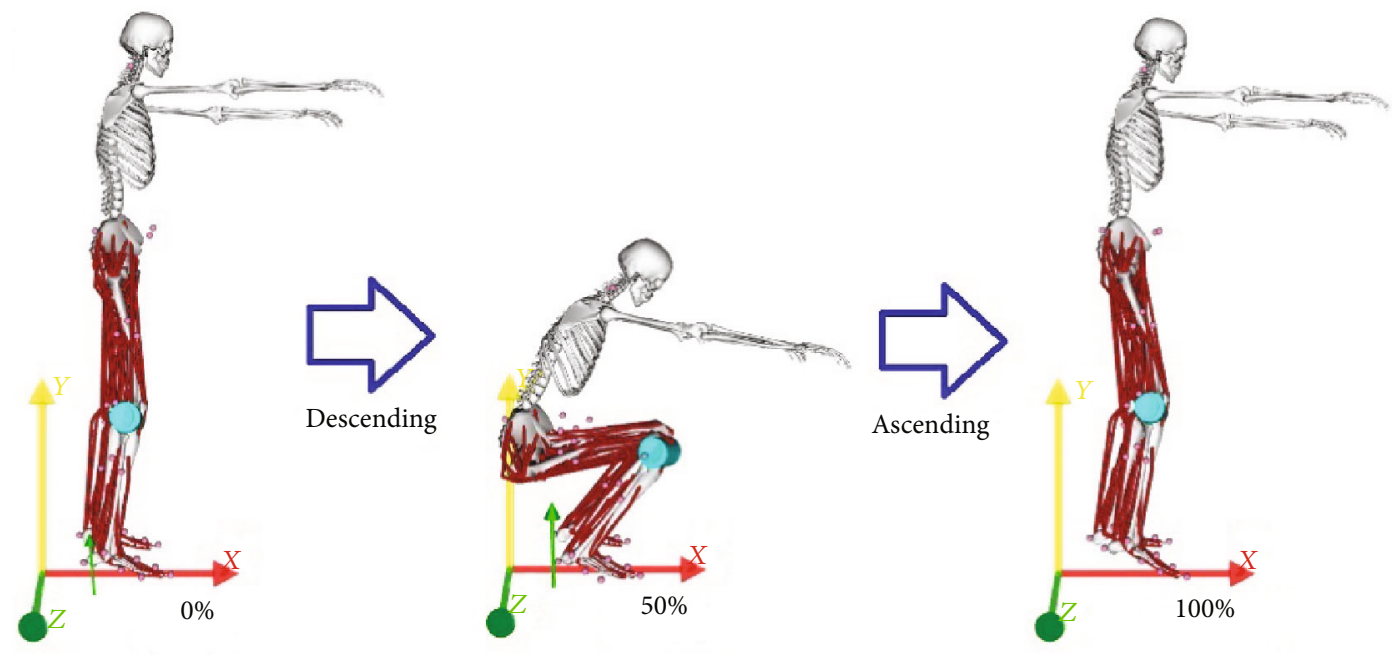

(b)

FIGURE 1: Illustration of model scaling (a) and squat descending and ascending phases (b).

for left and right side-specific. In specifics, the scale factors including torso (left/right acromion-ASIS), pelvis (left/right ASIS-sacrum), right femur (right ASIS-lateral right knee), right tibia (lateral right knee-lateral right malleoli), right foot (right calcaneus-right toe tip), left femur (left ASIS-lateral left knee), left tibia (lateral left knee-lateral left malleoli), and left foot (left calcaneus-left toe tip) were used. The static pose weights for each marker was manually adjusted as per the root mean square (RMS) error values (less than 0.002) between the experimental marker and virtual marker in the model. Secondly, the Inverse Kinematics algorithm was conducted to calculate the joint angles with minimized errors between experimental marker trajectories with virtual markers, followed by the third step of Inverse Dynamics to compute the joints moments. The next step Static Optimization was employed with a minimized sum of squared muscle activation to estimate the muscle force and activation $[17,19$, $21,22]$, as muscle force being a main factor contributing to joint loading. The Joint Reaction analysis was performed to compute the resultant forces from all relevant muscle forces, external loads, and inertial loads $[22,23]$ at the hip, knee, and ankle joints in the anterior-posterior $(x)$ (ant-post), superiorinferior $(y)$ (axial) and medial-lateral $(z)$ (med-lat) directions (global coordinates being illustrated in Figure 1). The threedimensional vectors of contact forces in the hip, knee, and ankle were set to apply on the Child (body) and expressed in the Child (frame) during joint reaction analysis, which were femur, tibia, and talus, respectively. A further Body Kinematics analysis was performed to calculate the Centre of Mass (CoM) trajectory for each model to quantify the squat depth (in $y$ vector), which was then normalized to the leg length for comparison.

2.4. Data Process and Statistical Analysis. This study mainly analysed the squatting stance from the right foot side stepping on the force platform to the right foot stepping off the 
force platform. All trials were processed and cropped from the right foot contact to stepping off the platform, with the threshold of vertical ground reaction force at $20 \mathrm{~N}$. The squatting stance was further divided into two phases [16], which were from the foot-on force platform and descending to the deepest position $(0 \sim 50 \%)$ and from the deepest position and ascending to the foot-off force platform (51 100\%) (Figure 1(b)). Joint angles and moments included hip flexion/extension, adduction/abduction, internal/external rotation, knee flexion/extension, and ankle dorsi/plantar flexion. The ROM in the hip, knee, and ankle were computed to illustrate joint flexibility. Joint moments were normalized by body mass $(\mathrm{Nm} / \mathrm{kg})$, and joint contact forces were normalized by body weight (\%BW).

The data process and statistical analysis were performed in MATLAB R2018a (MathWorks, MA, United States). Due to the one-dimensional (1D) time-varying features of the joint angles, moments, and contact forces, the data from one squatting stance was firstly interpolated to a dataset with 101 data points using cubic B-spline, followed by the averaged waveform of three trials from each participant. The open-source 1D Statistical Parametric Mapping (1dSPM) was based on Random Vector Field Theory to check data variability. The 0dSPM was used to check the significance of the discrete value, such as the Range of Motion (RoM) and squat depth. The independent-sample $t$ test package in the 1dSPM was used for the statistical analysis, with a significance level of $p<0.05$.

\section{Results}

3.1. Model Validation. The model validation approach consisted of three parts. Firstly, the peak joint angles at the hip (Caucasian: $108.5 \pm 12.9^{\circ}$ versus Asian: $110.7 \pm 7.8^{\circ}$ ) and knee (Caucasian: $119.3 \pm 23.4^{\circ}$ versus Asian: $139.9 \pm 4.9^{\circ}$ ) were in the range of $138^{\circ}$ (hip) and $145^{\circ}$ (knee) [16], respectively. Secondly, the results of joint angles, moments, and contact forces were compared against recent squatting studies. Lastly, the estimated muscle activation was compared against the measured surface EMG activities, presented in Figure 2.

3.2. Joint Angles and Squat Depth. The comparison of joint ROM and squat depth are presented in Table 1. A significant larger knee flexion-extension ROM was observed $(p=0.024)$. A significant deeper squat depth was found in the Asian individuals, while comparing to the Caucasian individuals $(p=0.028)$.

In terms of the comparison of time-varying joint angles during the squat, differences were found in the hip rotation that Asian exhibited larger external rotation during descending $(p=0.038,31-37 \%)$ and ascending $(p=0.04,62-66 \%)$ phases and in the ankle dorsiflexion that Asian dorsiflexed significantly during $43-46 \% \quad(p=0.045)$ and $67-74 \%$ $(p=0.026)$ of the squatting stance (Figure 3$)$.

3.3. Joint Moments. Differences in the joint moments exhibited that Asian had greater hip external rotation moment during $32-86 \%(p<0.001)$ of the squatting stance. Significantly greater knee flexion moments were also found in
Asian during $1-54 \%(p<0.001)$ and $63-100 \%(p<0.001)$ of the squatting stance. However, the Caucasian exhibited a larger dorsiflexion moment while standing up (95-97\%, $p=0.044$ ) during dynamic squatting (Figure 4).

3.4. Joint Forces. Hip joint contact forces of Asian differed from Caucasian in the ant-post direction during $2-12 \%$ $(p<0.001), 21-28 \%(p=0.004)$, and $95-100 \%(p=0.009)$ of the squatting stance. The differences in the axial direction are during $1-8 \%(p=0.002)$ and $99-100 \%(p=0.03)$ of the squatting stance, and differences in the med-lat direction are during $3-9 \%(p=0.022)$ and $96-99 \%(p=0.019)$ of the squatting stance (Figure 5).

Knee joint contact forces showed no significant difference between Asian and Caucasian in the ant-post direction. That of Asian differed from Caucasian in the axial direction during $1-2 \%(p=0.045), 7 \%(p=0.048), 9-33 \%(p<0.001)$, $36-37 \%(p=0.035), 40-48 \%(p<0.001), 67-87 \%(p<0.001)$, and $99-100 \%(p=0.039)$ of the squatting stance and in the med-lat direction during the whole squatting stance $(p<0.001)$ (Figure 5).

Ankle joint contact forces of Asian differed from Caucasian in the ant-post direction during 35-63\% $(p<0.001)$ of the squatting stance; in the axial direction during $35-62 \%$ $(p<0.001), 92-98 \%(p=0.003)$, and 100\% $(p=0.046)$ of the squatting stance; and in the med-lat direction during $42-54 \%(p<0.001)$ and $63-99 \%(p<0.001)$ of the squatting stance (Figure 5).

\section{Discussion}

This study presented a comparative investigation on the joint angles, moments, and contact forces in the lower extremity of the Asian and Caucasian individuals while performing the deep squat. A customized squat-specific musculoskeletal model was employed for the analyses, and the validation was performed via comparing the results of the current study to this model developer [16], previous squatting studies [6, 9, 24, 25], and OpenSim-estimated activation against EMG measured activities (Figure 2). It was found that Asian individuals presented greater external rotation in the hip and dorsiflexion at the ankle during the squat, descending, and ascending phases. A larger knee ROM was observed, although no significant difference exhibited in the timeseries angles between Asian and Caucasian individuals. Significantly larger hip rotation moment and knee extension moment were found in the Asian individuals while squatting. Differences in the hip contact forces were observed during the descending and ascending phases of the squat. The larger knee axial and med-lat contact forces were found throughout the squatting stance in the Asian individuals. However, the Caucasian individuals exhibited larger ankle ant-post and axial forces around the deepest squatting position, although larger ankle med-lat force was found in the Asian individuals.

The practice of the squat can enhance musculature functions and sports performances from the aspect of strength and conditioning [11]. The deep squat with external loading (such as barbell squat) was commonly practiced by professional weightlifting athletes and recreational amateurs [10, 

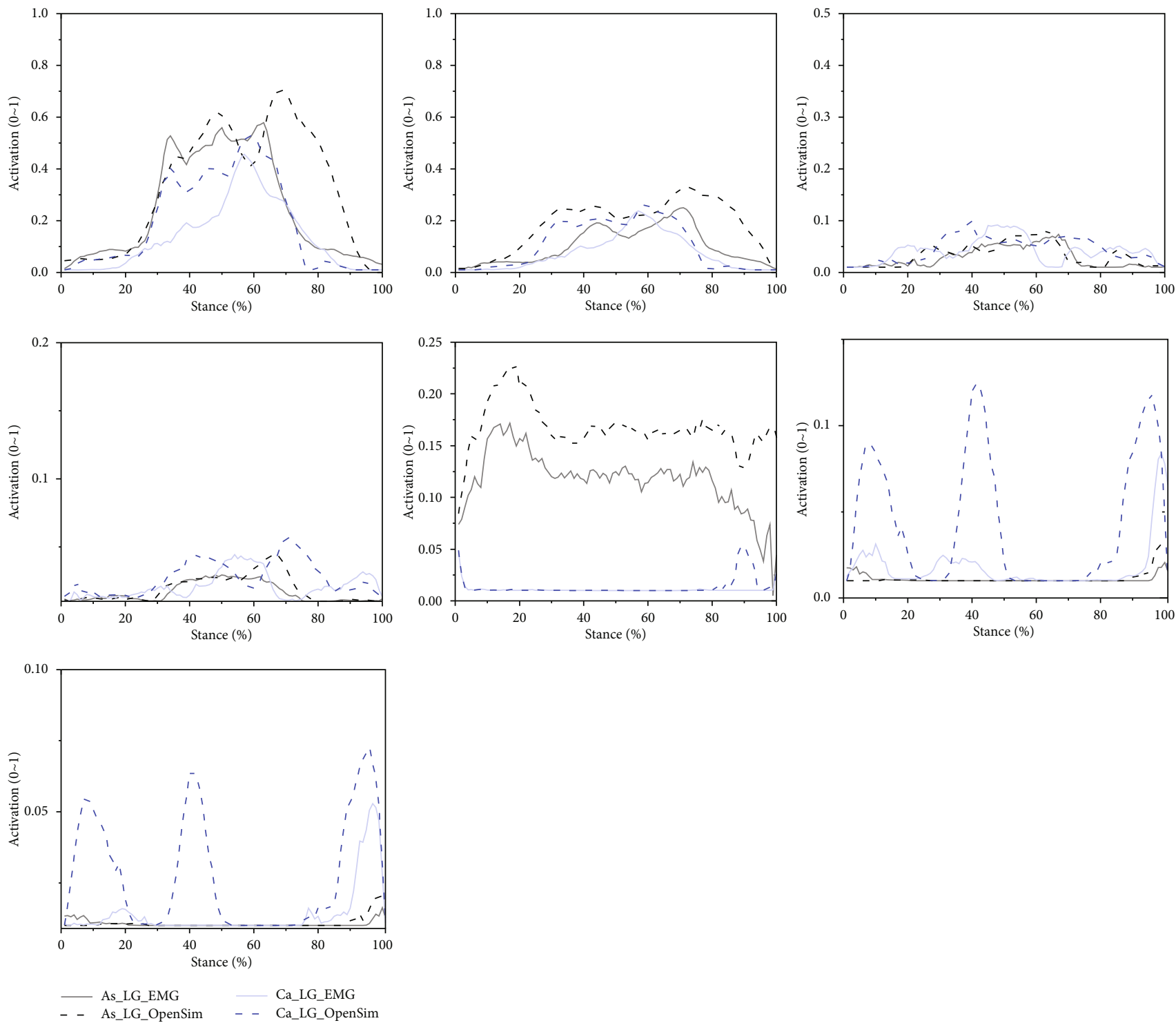

Figure 2: Comparisons of measured EMG activities against estimated (OpenSim) muscle activation in the Asian (As) and Caucasian (Ca) groups.

TABle 1: Hip, knee, and ankle joint range of motion during squat and the squat depth (\% leg length) in the Asian and Caucasian groups.

\begin{tabular}{lccccccccccccc}
\hline & \multicolumn{3}{c}{ Hip } & \multicolumn{3}{c}{ Knee } & \multicolumn{3}{c}{ Ankle } & \multicolumn{3}{c}{ Squat depth } \\
& Asian & Caucasian & $p$ & Asian & Caucasian & $p$ & Asian & Caucasian & $p$ & Asian & Caucasian & $p$ \\
\hline \multirow{2}{*}{ Sagittal } & 100.56 & 97.12 & & & 130.58 & 114.6 & \multirow{2}{*}{$0.024^{*}$} & 41.51 & 37.48 & 0.1 & 48.56 & 56.33 & $0.028^{*}$ \\
& $(6.62)$ & $(17.72)$ & 0.27 & $(4.98)$ & $(24.6)$ & & $(7.68)$ & $(7.14)$ & & $(2.73)$ & $(10.46)$ & \\
Coronal & 14.78 & $15.5(3.3)$ & 0.32 & - & - & - & - & - & - & - & - & - \\
Horizontal & $(4.11)$ & $31.1(8.8)$ & $29.8(11.1)$ & 0.38 & - & - & - & - & - & - & - & - \\
\hline
\end{tabular}

*Significant difference found between Asian and Caucasian $(p<0.05)$.

26]. Comprehensively revealing the biomechanical characteristics would provide implications to help increase performances and prevent potential injuries [4, 11, 27]. The influence of the foot placement and orientation; knee, hip, and pelvis motions; and spine and arm postures on the squat- ting performance was investigated previously using different techniques since the squat synchronously involved motor complexity in a close kinetic chain $[6,28]$.

In the current study, the Asian individuals exhibited greater hip external rotation angle and moment comparing 

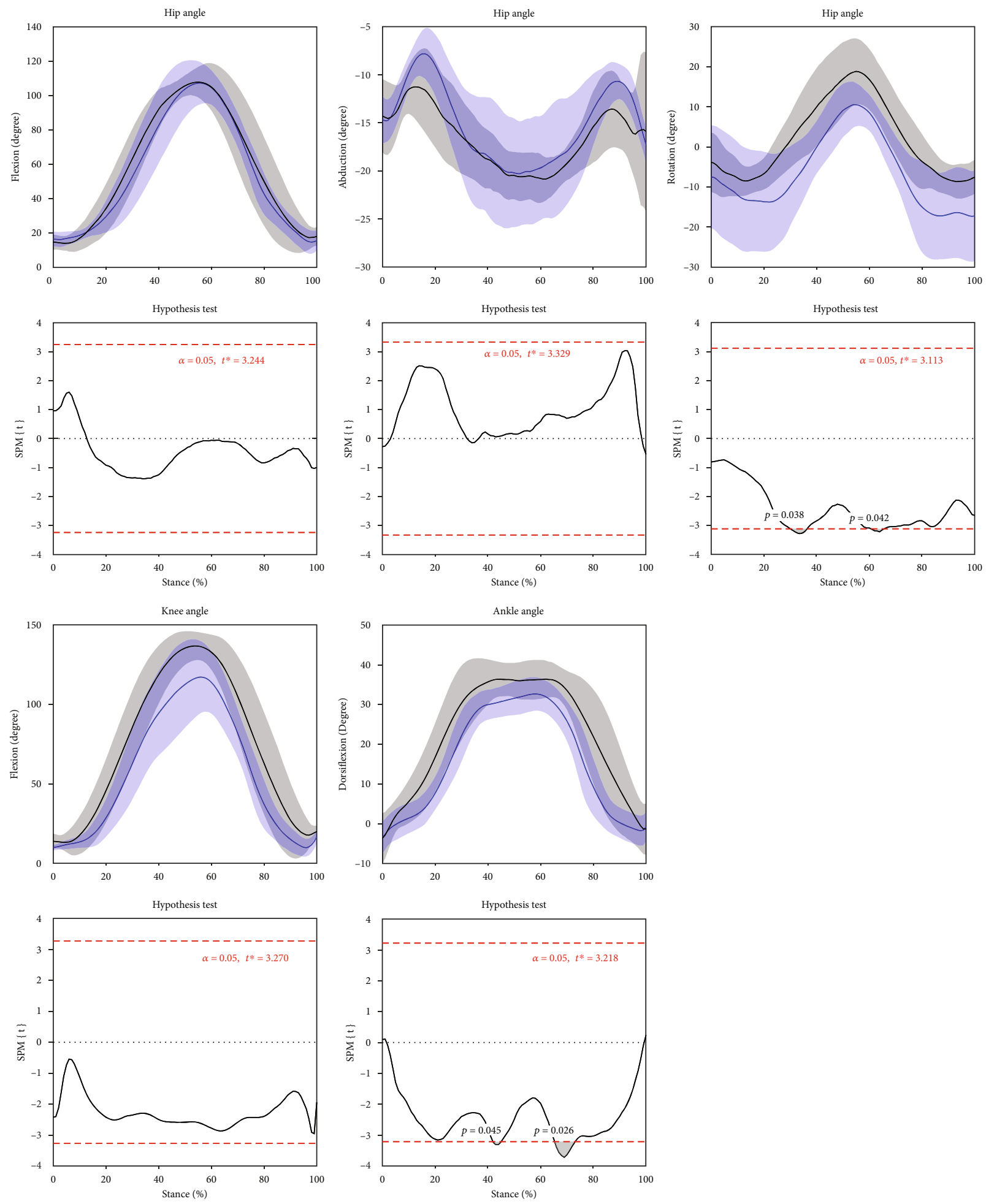

$\underset{\mathrm{SD}}{\text { Caucasian }} \quad$ Asian

FIGURE 3: Comparisons of joint angles using 1dSPM with highlighted significance.

to the Caucasian individuals, with the Caucasian individuals presenting similar hip ROM with a recent study on European Caucasian [4]. The nonsignificant difference in the external rotation may link with the coupled pelvis-trunk position as previously reported that pelvis tilting may affect hip rotation
[29]. An additional explanation could be that hip flexibility was correlated with squat depth, typically in Asian populations [27]. Due to the great hip rotation in the deep squat position, a significantly larger hip moment was observed in the Asian individuals in the current study. Furthermore, this 

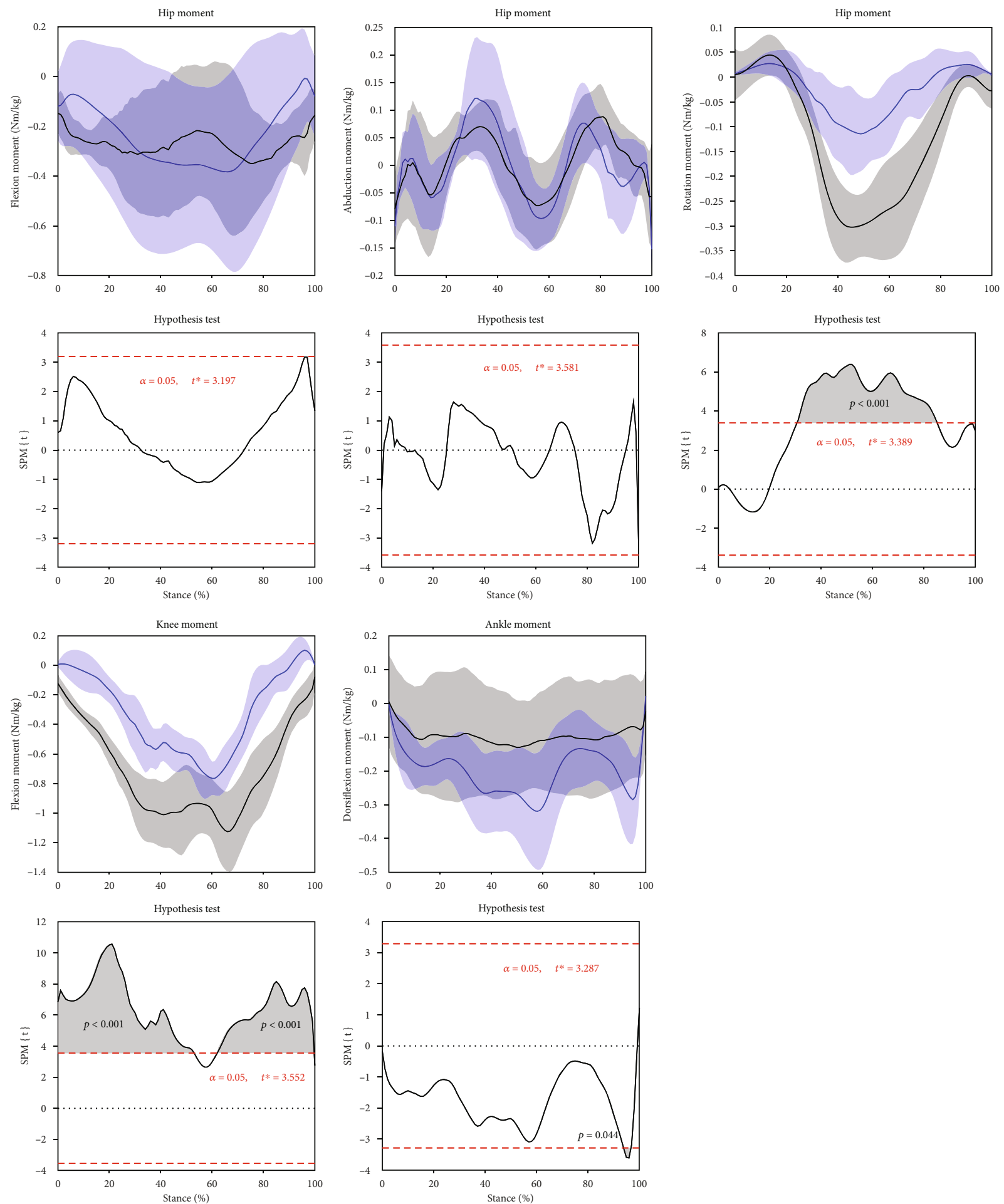

$$
\begin{aligned}
& \text { Caucasian } \\
& \mathrm{SD} \\
& \mathrm{S} \text { Asian } \\
& \mathrm{SD}
\end{aligned}
$$

FIGURE 4: Comparisons of joint moments using 1dSPM with highlighted significance.

study computed the hip contact forces via estimation from the joint reaction analysis during the squat. Interestingly, no significant difference was observed around the deepest squat position; however, differences were found during the initial, descending, and final ascending phases of the squat. The reason may be that the two groups performed the deep squat with different movement patterns and strategies [1], and we just instructed all participants to perform the deep 

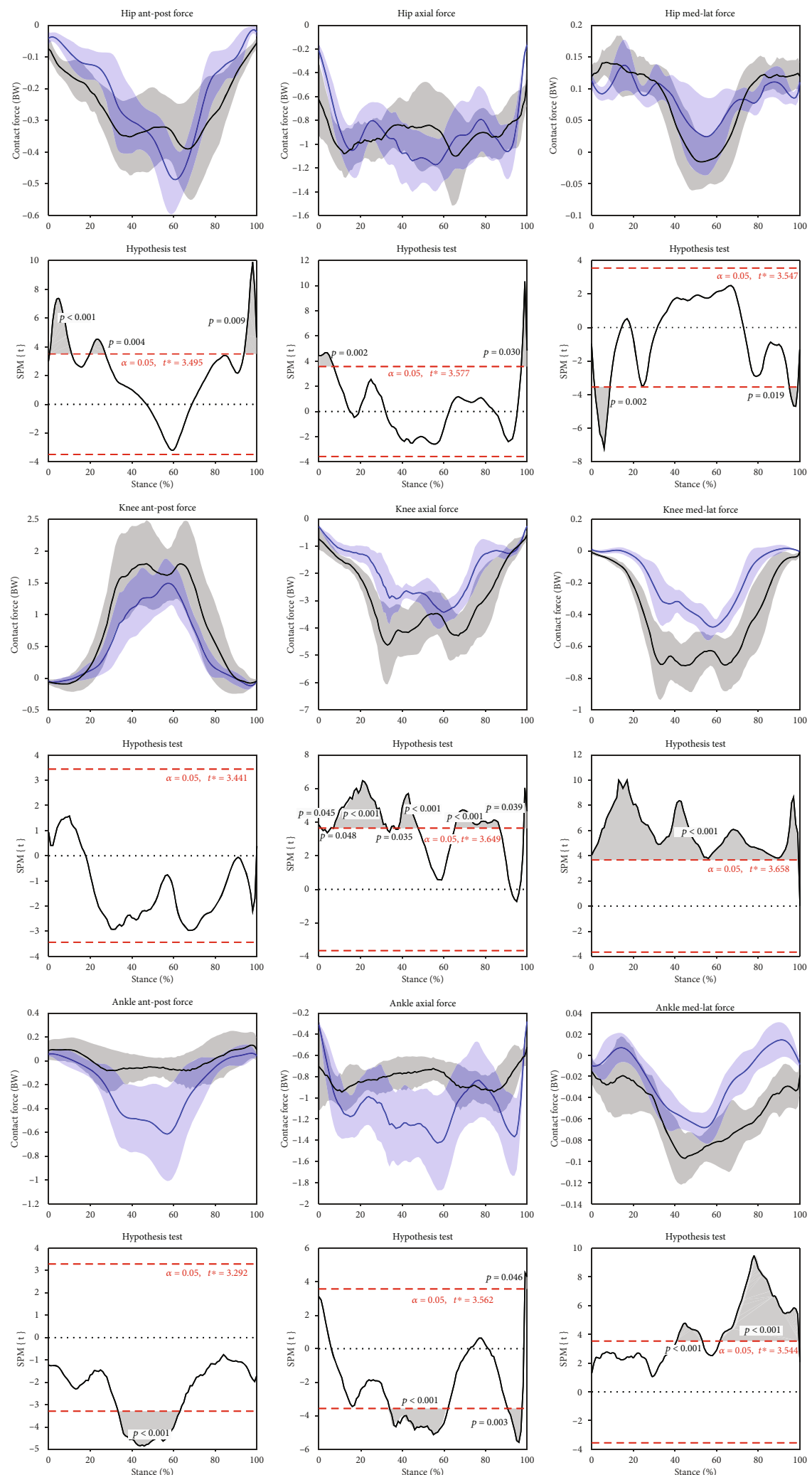

$$
\underset{\text { SD }}{\text { Caucasian }} \underset{\text { Asian }}{ }
$$

FIGURE 5: Comparisons of joint contact forces using 1dSPM with highlighted significance. 
squat with their self-preferred movement patterns in the current study.

Knee biomechanics has attracted extensive research interest due to its complex structure and highly reported injuries in the squat $[5,11]$. The time-series knee angles showed no significant difference between the two groups, while the Asian individuals presented a significantly larger ROM. It may be firstly due to the fact that participants performed the squat with their self-preferred patterns; thus, the individualized deepest squat positions were achieved $[1,27]$. Secondly, it may be due to the fact that the factors, such as squatting stance width, foot orientation, and squatting speed, were not controlled, which were previously being reported to affect the squat performance $[4,25]$. However, the larger knee flexion ROM in the current study was consistent with a previous study in Asian populations with greater squatting depth [27]. Therefore, the larger knee flexion moment of the Asian individuals in the current study was observed, similar to the pronounced greater knee flexion moment in a walk-squat study [14]. Additional findings of the knee axial and med-lat contact force differences may be evidence of loadings concentrated on the knee joint in the Asian group, as suggested by Thambyah [14] that repetitive descending and ascending motions may accumulate loadings on the soft tissue in the knee joint complexity. Additional findings of the estimated and measured muscle activation (Figure 2) may be another factors contributing higher knee loading for this study, as the joint reaction analysis calculated the resultant forces from all relevant muscle forces, external loads, and inertial loads [22, 23].

The foot is working as the interface of the kinetic chain while squatting, and the ankle joint ROM would affect the squat performance [24]. As found in the current study, the Asian individuals presented larger dorsiflexion around the deepest squat position. Interestingly, no difference in the ankle moment was observed, which might be that both groups performed the squat with comfortable movement patterns. However, the highly concentrated ant-post and axial contact forces in the ankle joint of the Caucasian individuals should be noted in particular, which are consistent with higher activated medial and lateral gastrocnemius muscles compared to Asian individuals. These may suggest that the muscles in the shank contributed predominantly to ankle joint contact forces while performing the deep squat, although no moment difference was found. Another previous study that reported that limiting ankle-dorsiflexion ROM could activate calf muscles activity could be a key evidence for the circumstance [24]. The higher med-lat contact force in the Asian individuals during the ascending phase might be the redirected foot orientation as observed highly activated tibia anterior muscle.

Several limitations should be accounted in the current study. Firstly, the squatting patterns, such as the stance width and foot orientation, were not unified across the participants, since the aim was to evaluate the difference in deep squat between Asian and Caucasian individuals without any manipulation. Secondly, the knee and ankle joints were modelled as a hinge joint (flexion/extension) with only sagittal ROM. This is due to the fact that the only available musculoskeletal models used in the current study were not profiled with other two degrees of freedom (coronal and horizontal).
Lastly, it should be noted that there might be a gender difference in the squat performance, as average squatting depth in Asian female (50.84\%) versus male (47.8\%) participants and Caucasian female (65.79\%) versus male (53.18\%) participants. However, the unequal number of male and female participants in the Asian and Caucasian groups may present hormonal changes [30], which need to be considered in the future studies as well.

\section{Conclusions}

This study evaluated the lower extremity biomechanics in the Asian and Caucasian individuals while performing the maximal squat using a customized squat-specific musculoskeletal model. The key findings of the current study were that, firstly, the greater knee contact forces were reported in the Asian individuals and, secondly, the greater ankle contact forces in the Caucasian individuals were notable while performing the deep squat comparing to the Asian cohorts. Knowledge found in the current study may provide implication for exercise practitioners and physiotherapists while designing schemes for the prevention of loading accumulation in the lower extremity.

\section{Data Availability}

The data for this study are available on reasonable request to the corresponding authors.

\section{Conflicts of Interest}

The authors declare that they have no competing interests.

\section{Acknowledgments}

The study was supported by the Zhejiang Pharmaceutical College and Research Academy of Grand Health in Ningbo University. We would like to acknowledge all participants in the test.

\section{References}

[1] A. Knox, N. Sculthorpe, J. S. Baker, and F. Grace, "Strength adaptation to squat exercise is different between Caucasian and South Asian novice exercisers," Research in Sports Medicine, vol. 25, no. 3, pp. 373-383, 2017.

[2] D. M. Ditchen, R. P. Ellegast, T. Gawliczek, B. Hartmann, and M. A. Rieger, "Occupational kneeling and squatting: development and validation of an assessment method combining measurements and diaries," International Archives of Occupational and Environmental Health, vol. 88, no. 2, pp. 153-165, 2015.

[3] Y. Zhang, D. J. Hunter, M. C. Nevitt et al., "Association of squatting with increased prevalence of radiographic tibiofemoral knee osteoarthritis: the Beijing Osteoarthritis Study," Arthritis \& Rheumatism, vol. 50, no. 4, pp. 1187-1192, 2004.

[4] S. Lorenzetti, M. Ostermann, F. Zeidler et al., "How to squat? Effects of various stance widths, foot placement angles and level of experience on knee, hip and trunk motion and loading," BMC Sports Science, Medicine and Rehabilitation, vol. 10, no. 1, 2018. 
[5] R. F. Escamilla, "Knee biomechanics of the dynamic squat exercise," Medicine and Science in Sports and Exercise, vol. 33, no. 1, pp. 127-141, 2001.

[6] W. R. Taylor, P. Schütz, G. Bergmann et al., "A comprehensive assessment of the musculoskeletal system: the CAMS-Knee data set," Journal of Biomechanics, vol. 65, pp. 32-39, 2017.

[7] C. D. Fregly, B. T. Kim, Z. Li, J. K. De Witt, and B. J. Fregly, "Estimated muscle loads during squat exercise in microgravity conditions," in ASME 2012 Summer Bioengineering Conference, Parts $A$ and B, pp. 367-368, Fajardo, Puerto Rico, USA, 2012.

[8] M. Kritz, J. Cronin, and P. Hume, "The bodyweight squat: a movement screen for the squat pattern," Strength and Conditioning Journal, vol. 31, no. 1, pp. 76-85, 2009.

[9] S. Almosnino, D. Kingston, and R. B. Graham, "Three-dimensional knee joint moments during performance of the bodyweight squat: effects of stance width and foot rotation," Journal of Applied Biomechanics, vol. 29, no. 1, pp. 33-43, 2013.

[10] H. Hartmann, K. Wirth, and M. Klusemann, "Analysis of the load on the knee joint and vertebral column with changes in squatting depth and weight load," Sports Medicine, vol. 43, no. 10, pp. 993-1008, 2013.

[11] B. J. Choenfeld, "Squatting kinematics and kinetics and their application to exercise performance," Journal of Strength and Conditioning Research, vol. 24, no. 12, pp. 3497-3506, 2010.

[12] E. Dooley, J. Carr, E. Carson, and S. Russell, "The effects of knee support on the sagittal lower-body joint kinematics and kinetics of deep squats," Journal of Biomechanics, vol. 82, pp. 164-170, 2018.

[13] J. Z. Wu, E. W. Sinsel, R. E. Carey, L. Zheng, C. M. Warren, and S. P. Breloff, "Biomechanical modeling of deep squatting: effects of the interface contact between posterior thigh and shank," Journal of Biomechanics, vol. 96, article 109333, 2019.

[14] A. Thambyah, "How critical are the tibiofemoral joint reaction forces during frequent squatting in Asian populations?," The Knee, vol. 15, no. 4, pp. 286-294, 2008.

[15] F. Schellenberg, W. R. Taylor, A. Trepczynski et al., "Evaluation of the accuracy of musculoskeletal simulation during squats by means of instrumented knee prostheses," Medical Engineering \& Physics, vol. 61, pp. 95-99, 2018.

[16] D. S. Catelli, M. Wesseling, I. Jonkers, and M. Lamontagne, "A musculoskeletal model customized for squatting task," Computer Methods in Biomechanics and Biomedical Engineering, vol. 22, no. 1, pp. 21-24, 2019.

[17] S. L. Delp, F. C. Anderson, A. S. Arnold et al., "OpenSim: opensource software to create and analyze dynamic simulations of movement," IEEE Transactions on Biomedical Engineering, vol. 54, no. 11, pp. 1940-1950, 2007.

[18] A. Rajagopal, C. L. Dembia, M. S. DeMers, D. D. Delp, J. L. Hicks, and S. L. Delp, "Full-body musculoskeletal model for muscle-driven simulation of human gait," IEEE Transactions on Biomedical Engineering, vol. 63, no. 10, pp. 2068-2079, 2016.

[19] Q. Mei, Y. Gu, L. Xiang, J. S. Baker, and J. Fernandez, "Foot pronation contributes to altered lower extremity loading after long distance running," Frontiers in Physiology, vol. 10, p. 573, 2019.

[20] M. Hall, L. E. Diamond, G. K. Lenton, C. Pizzolato, and D. J. Saxby, "Immediate effects of valgus knee bracing on tibiofemoral contact forces and knee muscle forces," Gait \& Posture, vol. 68, pp. 55-62, 2019.
[21] M. S. DeMers, S. Pal, and S. L. Delp, "Changes in tibiofemoral forces due to variations in muscle activity during walking," Journal of Orthopaedic Research, vol. 32, no. 6, pp. 769-776, 2014.

[22] Z. F. Lerner, D. J. Haight, M. S. DeMers, W. J. Board, and R. C. Browning, "The effects of walking speed on tibiofemoral loading estimated via musculoskeletal modeling," Journal of Applied Biomechanics, vol. 30, no. 2, pp. 197-205, 2014.

[23] Z. F. Lerner and R. C. Browning, "Compressive and shear hip joint contact forces are affected by pediatric obesity during walking," Journal of Biomechanics, vol. 49, no. 9, pp. 15471553, 2016.

[24] E. Macrum, D. R. Bell, M. Boling, M. Lewek, and D. Padua, "Effect of limiting ankle-dorsiflexion range of motion on lower extremity kinematics and muscle-activation patterns during a squat," Journal of Sport Rehabilitation, vol. 21, no. 2, pp. 144-150, 2012.

[25] R. Anderson, C. Courtney, and E. Carmeli, "EMG analysis of the vastus medialis/vastus lateralis muscles utilizing the unloaded narrow- and wide-stance squats," Journal of Sport Rehabilitation, vol. 7, no. 4, pp. 236-247, 1998.

[26] G. Liu, G. Fekete, H. Yang et al., "Comparative 3-dimensional kinematic analysis of snatch technique between top- elite and sub-elite male weightlifters in 69-kg category," Heliyon, vol. 4, no. 7, article e00658, 2018.

[27] S. H. Kim, O. Y. Kwon, K. N. Park, I. C. Jeon, and J. H. Weon, "Lower extremity strength and the range of motion in relation to squat depth," Journal of Human Kinetics, vol. 45, no. 1, pp. 59-69, 2015.

[28] G. Fekete, B. M. Csizmadia, M. A. Wahab, P. De Baets, L. V. Vanegas-Useche, and I. Bíró, "Patellofemoral model of the knee joint under non-standard squatting," DYNA, vol. 81, no. 183, pp. 60-67, 2014.

[29] J. J. Bagwell, T. Y. Fukuda, and C. M. Powers, "Sagittal plane pelvis motion influences transverse plane motion of the femur: kinematic coupling at the hip joint," Gait \& Posture, vol. 43, pp. 120-124, 2016.

[30] G. M. Cesar, V. S. Pereira, P. R. P. Santiago et al., "Variations in dynamic knee valgus and gluteus medius onset timing in nonathletic females related to hormonal changes during the menstrual cycle," The Knee, vol. 18, no. 4, pp. 224-230, 2011. 